\title{
Sarcopenia: From definition to treatment
}

\author{
Yannis Dionyssiotis, ${ }^{1}$ Athina Kapsokoulou, ${ }^{2}$ Eleni Samlidi, ${ }^{3}$ \\ Antonios G. Angoules, ${ }^{4}$ Jannis Papathanasiou, ${ }^{5}$ Efstathios Chronopoulos, ${ }^{6}$ \\ Ifigenia Kostoglou-Athanassiou, ${ }^{7}$ Georgios Trovas $^{8}$
}

\begin{abstract}
${ }^{1}$ Physical Medicine \& Rehabilitation Department, European Interbalkan Medical Center, Thessaloniki, Greece; ${ }^{2}$ Medical School of Patras, Rio, Patras, Greece; ${ }^{3}$ Medical School of Thessaly, Larissa, Greece; ${ }^{4}$ Department of Medical Laboratories, Technological Educational Institute of Athens, Athens, Greece; ${ }^{5}$ Department of Kinesitherapy, Faculty of Public Health, Medical University of Sofia, Bulgaria; ${ }^{6} 2$ nd Orthopaedic Department, Athens University; Konstantopoulio General Hospital; Athens, Greece; ' Endocrinology Department, General Hospital Asklepieio Voulas, Voula, Greece; ${ }^{8}$ Laboratory for Research of the Musculoskeletal System, University of Athens, Kifissia, Greece
\end{abstract}

\section{INTRODUCTION}

Over the last few decades, worldwide average life expectancy has grown significantly and therefore the percentage of the elderly has correspondingly increased. As a result, clinical medicine has focused much interest on the latter age group and their health problems. During the $20^{\text {th }}$ century, the typical elderly patient was a person with an acute or chronic disease but typically without any major disability. In contrast, the $21^{\text {st }}$ century older patient's profile is characterized by considerable comorbidity, including a great number of chronic conditions with frequent acute episodes, all of which has an adverse effect on functionality. Accordingly, while the health system once focused on treating exclusively the disease, it nowadays needs to give greater attention to the rehabilitation of disability. ${ }^{1}$

Key words: DXA, Drugs, Nutrition, Physical activity, Rehabilitation, Sarcopenia

Address for correspondence:

Yannis Dionyssiotis, Physical Medicine \& Rehabilitation

Department, European Interbalkan Medical Center,

Thessaloniki, Greece; E-mail: yannis_dionyssiotis@hotmail.com

Received: 04-12-2017, Accepted: 18-01-2017
Age-related muscle loss is one of the main characteristics of ageing. It appears more often in physically inactive people, although it also affects those who have been active throughout their lifetime, ${ }^{1}$ this indicating that while the modern sedentary lifestyle is contributive to this phenomenon, it is not the only causative factor. Numerous changes take place as we grow old: apart from the aforementioned increasing tendency towards a sedentary lifestyle, there are changes in hormone levels and in the body's protein demands as well as the unavoidable degeneration (death) of the motor neurons. ${ }^{2}$

\section{SEEKING A DEFINITION OF SARCOPENIA}

Sarcopenia, an age-related loss of muscle mass and power, has recently been recognized as a disease. It has been included in the $10^{\text {th }}$ edition of the ICD-10 (the International Statistical Classification of Diseases and Related Health Problems), in the categories "muscle wasting and atrophy not elsewhere classified" (code M62.5) and "disorder of muscle, unspecified" (M62.9), and since September 2016 it has been assigned code M62.84. The latter has constituted an important step towards the recognition of sarcopenia as a disease and, what is more, it is expected to increase the availability of diagnostic methods (diagnostic lab tests and trials) and to 
motivate the pharmaceutical companies to develop appropriate medical treatment. ${ }^{3}$

Despite its recognition as a disease, sarcopenia does not as yet constitute adequate indication for therapeutic intervention; thus, interest is focused on the circumstances and the condition under which such organizations as the FDA (Food and Drug Administration) could consider sarcopenia as an indication for treatment. ${ }^{4}$

Irwin Rosenberg, who coined the term 'sarcopenia' in 1989, meticulously investigated the disorder, particularly stressing the importance of loss of muscle mass in the process of increasing disability. He defined sarcopenia as the observed age-related decrease in muscle mass and the decrease of muscle strength and functionality that concurrently takes place. The definition has been steadily improved since 1989 as well as since the first Sarcopenia Workshop which took place in 1994 organized by the National Ageing Research Institute. Today, the definition of sarcopenia considers both the loss of muscle function (muscle power) and the loss of muscle quality (muscle strength/muscle mass unit), in other words, the increasing inability of muscle tissue to generate force in addition to the loss of muscle protein. It has not yet been clarified whether the loss of muscle function in the elderly (difficulties in e.g. rising from a chair, avoiding falls, walking in the street, carrying weights) is mainly the result of a decrease in muscle mass or a deterioration in the quality of muscle tissue. ${ }^{5}$

Generally speaking, low muscle mass is common among the elderly and numerous studies have been designed to determine the severity of the problem. Certain studies have examined the frequency of the disease among the population (male and female) of the USA (NMEHS, NHANES III, CHS, ABC REP) and others populations of Europe and Asia (INCHIANTI, EPIDOS, LASA, CHINESE). The results have varied, with the estimated prevalence ranging from $10 \%$ to $50 \%$ depending on the study and a discordance existing regarding the gender more at risk. Given that until recently an articulate mutual definition of sarcopenia had not been found and that the criteria of participation and elimination are likely to have been different in each study brings into question the credibility of the results and limits the possibility of generalization. In one reliable study, the prevalence of sarcopenia was dramatically increased along with age, from $4 \%$ in men and 3\% in women aged $70-75$ years old to $16 \%$ and $13 \%$, respectively, in persons aged 85 or more. ${ }^{6}$ It is nevertheless well established that with advancing age, muscle mass and power deteriorate and their decline below a certain level (cutoff) leads to disability. Certainly, the disability cutoff is affected by other factors. ${ }^{7}$

Moreover, it has been observed that patients suffering from both sarcopenia and osteoporosis were at a greater risk of falls, therefore the incidence of fractures in this cohort was higher. ${ }^{8}$ It is thus evident that the treatment of sarcopenia will considerably improve patients' wellbeing, especially if they are dealing with other diseases related to senility.

NHANES III (the National Health and Nutrition Examination Survey) has recorded the increasing frequency of sarcopenia with advancing age. However, in this study the description of sarcopenia was defined with no criteria other than muscle mass, whilst there is no information considering cachexia (due to other diseases), starvation (nutritional deficiency) and frailty (functional inability). ${ }^{9}$

As far as the definition of sarcopenia is concerned, it is usually complicated by the fact that there is an overlap between the diseases/conditions cachexia, sarcopenia, starvation and frailty. This conceptual confusion, found even among specialists, is due to the fact that all the abovementioned lead to muscle loss and are frequently related to each other. It is crucial that a clarification be made in clinical practice as different clinical and therapeutic treatment is required in each case. ${ }^{10}$

The formulation of an accurate definition will enable achievement of a more accurate diagnosis and of better designed clinical studies so that a conclusion may be drawn regarding the adequacy of the present therapeutic interventions. Just as for osteoporosis, a very frequent problem among elders, there are a number of medical options, there is likewise a need for drugs and lifestyle changes for the improvement of sarcopenia and of the functional ability of the elderly. Moreover, there are the financial costs that must be considered (incurred due to mobility impairment, 
the toxicity of some drug combinations, infections during hospitalization), the total exceeding the one of the osteoporotic fractures i.e. 18.5 versus 16.3 billion dollars, respectively. ${ }^{11}$

Because of the lack of a consistent operational definition, different parameters have been assessed and a variety of testing methods have been used by the many investigations into sarcopenia. More specifically, Baumgartner et al defined the presence or absence of sarcopenia according to muscle mass (ASM-appendicular skeletal muscle mass) measured via the DXA (dual energy X-ray absorptiometry) method (Figure 1). Patients were considered as suffering from sarcopenia whose ratio $\mathrm{ASM} /$ height $^{2}$ was less than 2 standard deviation (SD) compared to the expected normal value for the average young adult. The DXA scan has over the years provided an easy diagnostic method: however, it is not available in all health systems and, moreover, it does not evaluate functional ability. ${ }^{12}$

The European Working Group on Sarcopenia in Older People (EWGSOP) and the International

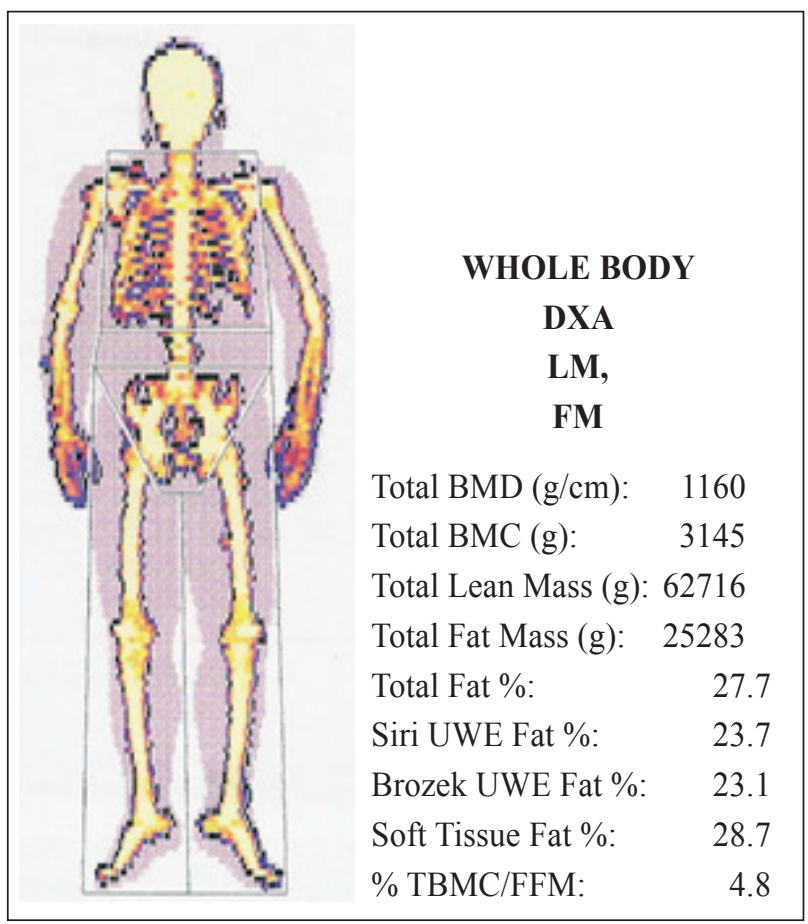

Figure 1. Control values of parameters using body DXA norland.
Sarcopenia Consensus Conference Working Group (ISCCWG) have pointed to the growing prevalence of the disease and have reviewed the scientific facts and the literature with regard to therapeutic intervention. ${ }^{13}$ EWGSOP defined sarcopenia as the syndrome characterized by a progressive and, eventually, total loss of skeletal muscle mass and strength, this being additionally associated with the risk of bodily and mental impairment, poor quality of life and early mortality. There are two criteria for diagnosing sarcopenia: low muscle mass + low muscle strength and low physical performance (decreased functionality) ${ }^{8}$ (Figure 2).

Using techniques such as DXA and BIA (Bioimpedance Analysis) and tests including grasping dynamometry and walking speed (gait speed test) or the SPPB test (Short Physical Performance Battery), the EWGSOP recommended that diagnosis be based on low muscle mass + low muscle function (strength or performance). Patients with less than 2 standard deviation (SD) compared to the expected normal value for the average young adult had sarcopenia. The main advantage of this definition is that it additionally provided information about muscle functionality. The EWGSOP categorized sarcopenia, according to the cause, into primary (or age-related) and secondary (when one or more non-age-related causes are evident) sarcopenia. Secondary sarcopenia has been further subdivided into sarcopenia related to: physical inactivity (e.g. after prolonged bed rest, low everyday/habitual physical activity, sedentary

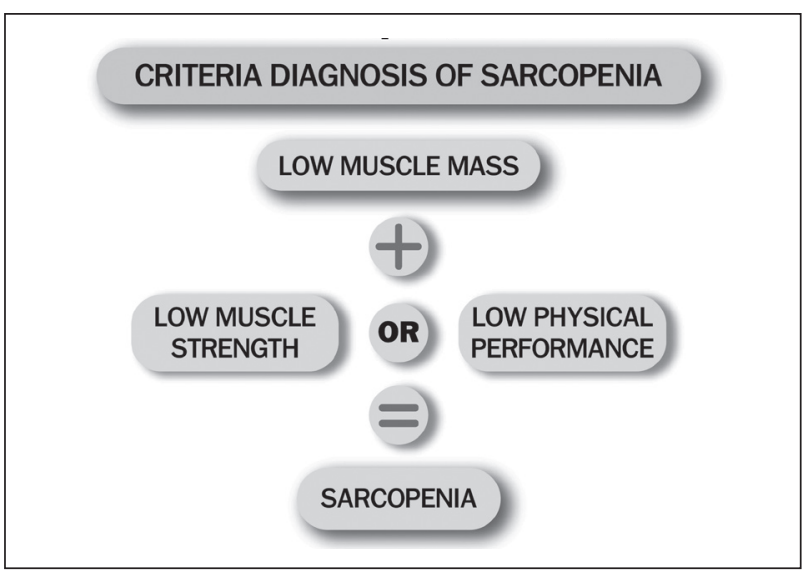

Figure 2. The European Working Group on Sarcopenia in Older People (EWGSOP). 
lifestyle), a particular disease (e.g. advanced organ failure, inflammatory diseases, malignancy, endocrinopathy) and lastly, nutrition (less-than-optimal diet, malabsorption, gastrointestinal disorders, druginduced anorexia) (Figure 3).

The ISCCWG's definition of sarcopenia was based on muscle mass and physical performance. After a meeting in Rome, ISCCWG published a Consensus that sarcopenia should be evaluated in older adults with clinically observed declines in physical functioning, activities of daily living, strength and health status, and those experiencing recurrent falls, recent weight loss, hospitalization or chronic conditions associated with muscle loss. ${ }^{14}$

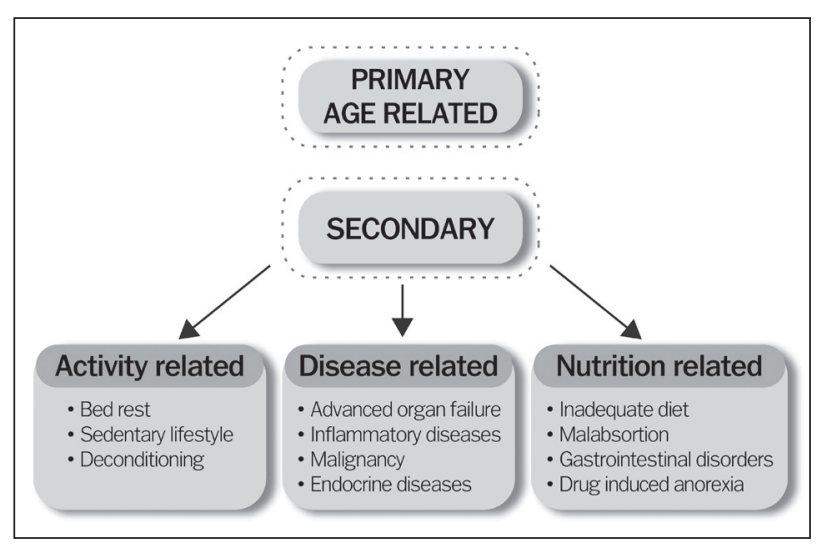

Figure 3. EWGSOP - Sarcopenia categories.

\section{PATHOPHYSIOLOGY OF PRIMARY (AGE-RELATED) SARCOPENIA}

The pathophysiology of sarcopenia in the elderly is complex with a plethora of internal and external procedures contributing to its development. As regards internal processes, the most important factors are the decrease of anabolic hormones (testosterone, estrogens, growth hormone, etc), the increased apoptotic activity of muscle fibers, the increase in preinflammatory cytokines (TNF-a, IL-6), oxidative stress due to accumulation of reactive oxygen radicals, the change in mitochondrial activity in muscle cells and the decrease in motor neurons. The external factors related to low functionality in the elderly include an insufficient intake of dietary protein resulting in loss of muscle mass and function, vitamin D deficiency, acute and chronic comorbidities leading to decreased physical activity and often to periods of prolonged immobilization and an age- and/or disease-related increased production of proinflammatory cytokines that induces proteolysis (cachexia). ${ }^{15}$

As a person ages changes take place in their protein metabolism since ageing affects systemic factors and hormones that are involved in the construction and deconstruction of proteins. For example, in elderly patients the observed decreased effectiveness of GH (growth hormone), IGF-1 (insulin-like growth factor-1) and insulin together with the concurrent increased activity of cytokines, catabolic hormone cortisol and myostatin adversely affect and inhibit protein biosynthesis. Myostatin is a hormone that acts on the myosatellite cells (precursors to skeletal muscle cells) and inhibits their growth and differentiation to mature muscle cells. ${ }^{16}$

The importance of muscle tissue for optimal functioning of the body is, from the biochemical point of view, enormous. ${ }^{17}$ The muscle system is the body's largest storage depot for protein, which in such conditions as stress and malnutrition furnishes constant amino acid supplementation so that protein synthesis may be continued in other basic tissues. Since the majority of glucose is stored in skeletal muscles, decreased muscle mass plays a major role in the disordered glucose metabolism of patients with insulin intolerance and diabetes mellitus type II. Moreover, given that skeletal muscle is the highest consumer of energy (chemical energy), thus positively contributing to basal metabolic rate (BMR), muscle loss in the elderly is the main cause of age-related decreased BMR and energy expenditure. The possible connection between low muscle mass and functionality and pathological glucose tolerance was reported in a publication by Hedman et al which showed that the association between the muscle fiber synthesis and glucose sensitivity though important is not strong $(\mathrm{r}=0.33) .{ }^{18} \mathrm{It}$ is known that glucose transporter 4 (GLUT 4) plays a major role in regulating glucose homeostasis, with intracellular glucose transport being triggered by insulin, muscle contractions or exercise. Meanwhile, care must be exercised with regard to identifying the mechanisms that cause loss of strength in the elderly. First, elderly persons who lead a sedentary 
lifestyle and at the same time have circulation and respiratory problems though also presenting "normal" mechanical muscle strength are probably suffering from a glucose muscle transport disorder. Second, there are thought to be patients who are being treated as diabetics but who are actually suffering from an undisclosed muscle disorder ${ }^{17}$

Old age comes with significant changes in muscle tissue architecture. There is a loss of motor units, due to the denervation of the fast twitch motor units, that include type II muscle fibers and, to a smaller degree, slow twitch motor units (with type I muscle fibers). After denervation, the muscle fibers can be recruited from the remaining motor units, at the same time changing their type, according to the muscle fiber type contained in the motor unit which recruits them. As a result, some denervated type II muscle fibers (which, when contracted, produce great power but for a short time) are turned into type I muscle fibers (which produce a smaller amount of power but last less time). Thus, with advancing age muscle tissue content in type I muscle fibers increases, while the number and mass of type II muscle fibers decreases (due to atrophy), which leads to a significant reduction in the maximum power that the muscle can produce. In elderly patients with sarcopenia, no recruitment takes place following muscle fiber denervation, which causes an even greater loss of motor muscle units, muscle atrophy and fraility. ${ }^{16}$ Another change that comes with age is related to the elasticity of the muscle-tendon system or muscle-tendon unit. The tendon, as the weakest link in the chain, is the final regulator of all the region's strength connections (muscle-bones). The tendons have an essential role in body movement since they transmit force between bones and muscles. As a result, age-related modifications in movement are intimately bound up with the tendon's mechanical properties, which, together with the muscles, exert an integrated effect. The loss of tendon elasticity with advancing age thus induces a decrease of muscle contraction capacity. ${ }^{16,19}$

\section{DIAGNOSIS OF SARCOPENIA}

Regarding the diagnosis of sarcopenia, it must be specified, in accordance with the definition and using adequate measurements and tests, whether there is a loss of muscle mass, a decrease of muscle power or a disorder of functionality and physical performance. ${ }^{8}$

Determination of muscle mass can be made using anthropometric methods (height, weight and other body measurements), which are technically easy to carry out and low-cost but lack reliability. In addition, biological methods may be used (creatinine secretion, whole body potassium using radioisotope ${ }^{40} \mathrm{~K}$ ), which are more complex but more dependable. Lastly, imaging methods can be used (MRI, CT-scan) which, despite their high cost, the difficulty in performing them and the small exposure to radiation, provide valuable information about muscle quality. Analysis of body composition and determination of muscle mass percentage can be achieved using surface electrodes and via the bio-impedancemetry method, which is easily applied and low-cost, albeit not entirely reliable. DXA appears to be quite useful as it has a low cost, is easy to use and is also reliable. For the diagnosis of sarcopenia, measurement of appendicular muscle mass (ASM), i.e. of the skeletal muscle mass of the four limbs, needs to be taken ${ }^{20}$ (Figure 4).

DXA systems provide a measurement of appendicular lean soft tissue (ALST), which is a fat and bone mineral-free tissue including muscle and other components such as skin, tendons and connective tissue. SM constitutes the largest fraction of ALST. Ap-

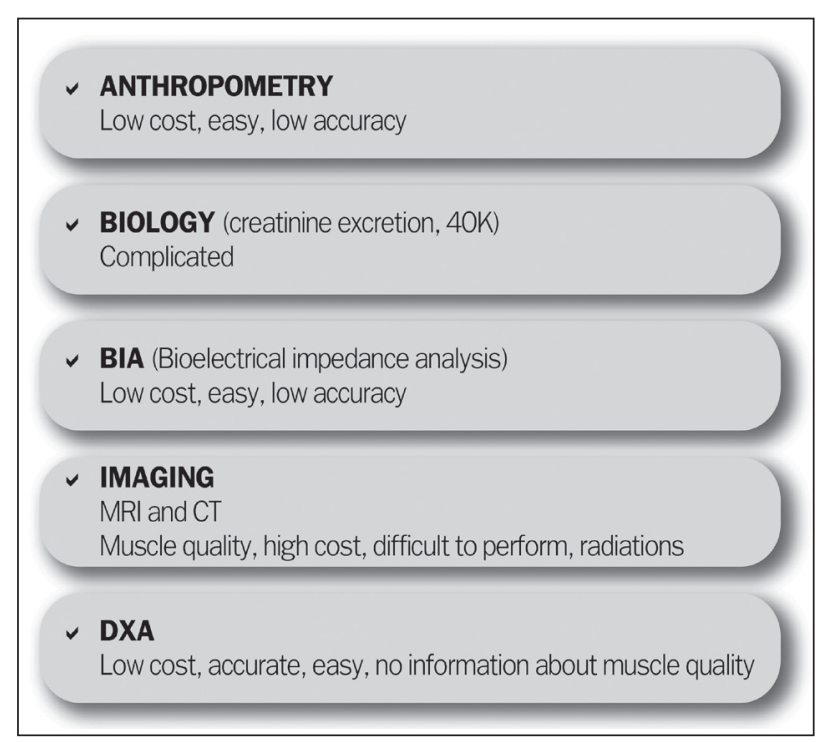

Figure 4. Muscle mass assessment tools. 
pendicular skeletal muscle mass (ASM), as measured by DXA, is quite helpful in diagnosing sarcopenia. ${ }^{21}$

In the Rosetta study examining the frequency of sarcopenia in an elderly population in New Mexico the diagnosis of sarcopenia was based on the skeletal muscle mass index (SMI), which is the ASM to the height ${ }^{2}$ ratio $\left(\mathrm{SMI}=\mathrm{ASM} /\right.$ height $^{2}$, counting units: $\mathrm{kg} /$ $\mathrm{m}^{2}$ ). Indicatives of sarcopenia were considered to be the SMI values which were less than 2 standard deviations (SD) below the mean value of a young population's SMI. More specifically, the limit for men was 7.26 $\mathrm{kg} / \mathrm{m}^{2}$ and for women $5.45 \mathrm{~kg} / \mathrm{m}^{2}$. The strategy used to diagnose sarcopenia was similar to that used for osteoporosis. To set the diagnosis of osteoporosis, superficial bone density $\left(\mathrm{gr} / \mathrm{cm}^{2}\right)$ measured by DXA (gold standard for the diagnosis of osteoporosis) is defined by the T-score, the number of standard deviations of the patient's value in comparison to mean bone density value of a normal young adult of the same gender and race. Thus, if the patient's T-score is less than 2.5 standard deviations from the reference value, the patient is suffering from osteoporosis. The 2.5 standard deviation cut-off was chosen based on the observed fracture risk (specific hip fracture risk). For a diagnosis of sarcopenia, muscle mass is measured and subsequently the T-score is calculated. ${ }^{12}$

For the purpose of determining the quantitative relationship between muscle mass and disability (decreased physical performance and functionality), Janssen et al studied 4,449 individuals who participated in the NHANES study during the period 1988-1994. Using the SMI as an indicator for the diagnosis of sarcopenia they came to the following conclusions: people with a SMI value of 8.51-10.75 in men and 5.75-6.75 in women were at medium risk for disability, whilst those with an even lower SMI (less than 8.50 in men and 5.75 in women) were at a greater risk, generally showing a higher risk of physical disability. ${ }^{9}$

However, they found an increased physical disability risk in women with very high SMI values. The increased physical disability risk in women with very high SMI values may have in part reflected the increased fat mass and obesity in these subjects. Fat mass is an independent predictor of physical disability and fat mass was considerably higher (39.2 kg vs. $28.1 \mathrm{~kg}$ ) in women with very high SMI values $\left(\geq 9.00 \mathrm{~kg} / \mathrm{m}^{2}\right)$ than in women with moderately high SMI values $\left(6.75-8.99 \mathrm{~kg} / \mathrm{m}^{2}\right){ }^{9}$

To conclude, using the SMI to define sarcopenia has some significant advantages, including that it is convenient, quick and easy to apply. However, there are a few serious disadvantages. These include the need to use DXA, which might not be available in every center, the definition of the threshold criteria which often differ, not taking into account the person's body weight and whether they are thin or obese (for example, thin people may have a low muscle mass without having any mobility limitations, while obese persons with generally a high muscle mass, but still lower in comparison to their body weight, may experience mobility limitations) and, finally, the fact that while focusing on muscle mass, a crucial parameter for the definition of sarcopenia, it does not take into consideration the strength that is produced per muscle mass unit and which differs greatly between the two genders and steadily declines with age.

On the other hand, it has been shown that apart from muscle mass (especially of the low limbs), muscle power independently defines the degree of motor impairment. Nevertheless, these two parameters are related, as a strong connection between lower limb muscle mass and muscle power has been found $(\mathrm{r}=0.78$, $\mathrm{p}<0.01$ ), while muscle mass is responsible for $60 \%$ of the total change in muscle power. Adequate muscle strength and power depend on multiple factors and thus additionally demand good functioning of the cerebral motor cortex, an intact spinal cord, normal conduction of electrical impulses, normal muscle mass and muscle tissue architecture, low density of myocytes in fatty acids and a normal stimulationcontraction junction. ${ }^{22}$

The next step in the diagnostic procedure is the use of tests that evaluate physical performance or muscle strength (possibly also power). ${ }^{23}$ To assess physical performance, some tests are used such as the chair stand test (sitting-rising test), the walk test (walking test), the functional reach test (achievable functionality test) and the modified Romberg test. Another test to evaluate static and dynamic balance is the Timed Up and Go Test (TUG) during which the patient stands up from the chair, walks 3 meters, turns round, walks back to the chair and sits 
down again. This test assesses only basic everyday moves, such as rising, walking, turning and sitting but not walking over an obstacle, which can cause slips and falls.

In general, some of the tests and measurements that can reveal limitations in functionality and physical activity caused by sarcopenia are the following: knee extension isometric torque, grasping power, lower limb muscle strength, 4- and 6-minute walking tests, the Short Physical Performance Battery (SPPB) that scores walking and balancing performance, the rising from a chair test and, lastly, the one-leg balance test.

The measurement of muscle power can be made with isometric methods, although these are static measurements, as they do not reproduce normal movement, or with isokinetic methods, which have the limitation of making the movement at specific angular velocities, which is a situation that is far from normal.

When measuring performance, the movements must be described by terms that include force, velocity and acceleration. Force $(\mathrm{N})$ causes acceleration (a). Every movement is caused by the action of a force at some time, thus it should be measured as Power (W) (force $\mathrm{x}$ distance $=$ work, work/time and force $\times$ velocity $=$ power $).{ }^{24}$

Two tests, jumping mechanography and rising from a chair, measure the power during that activity. Jumping mechanography can be used in very frail people who are not able to get up from a chair. The evaluation of muscle quality is made with measurements that evaluate muscle mass and functionality and includes definition of muscle mass and assessment of functionality through a series of tests such as grip strength, knee extension power and muscle power. These tests, designed to identify patients with motor problems (and presenting some small differences in sensitivity and specificity), can be easily performed in the clinical setting, while they are also readily available and quite simple as they do not require any devices. Nevertheless, their validity is limited due to the fact that the normal threshold is not strictly defined and the patient's performance depends on such factors as motivation, will and pain (for example, a patient with osteoarthritis), and not only on muscle mass, power and strength. ${ }^{25}$
In order to complete a patient's investigation their biochemical profile needs to be assessed, including inflammatory markers (CRP, interleukin-6, TNF-a), clinical-pathological markers (hemoglobin, albumin, urine creatinine), hormone levels (testosterone, insulinlike growth factor-1, vitamin D). ${ }^{26}$

\section{PREVENTION-TREATMENT-REHABILITATION: BASIC PRINCIPLES AND FUTURE TARGETS}

\section{Nutrition}

One of the interventions for the prevention and treatment of sarcopenia is nutrition. Malnutrition is responsible for numerous health problems, one of which is the loss of muscle mass. Older adults often have reduced food intake and therefore are vulnerable to malnutrition. ${ }^{27,28}$ Especially for someone who already suffers from sarcopenia, malnutrition can cause protein deficiency, further worsening the loss of muscle mass. An American survey showed that in the over 50 years age group, $32-41 \%$ of women and $22-38 \%$ of men consume less than the recommended daily protein intake. ${ }^{29}$ Meanwhile, a 3-year study showed that protein intake maintained muscle mass in women and men aged 70-79. People with the highest daily dietary protein intake at the time of this study lost $40 \%$ less muscle mass than those with the lowest intake. ${ }^{30}$ It has in addition been found that diets rich in acid-producing foods (such as meat and cereal grains) and poor in food that do not produce acid (fruit and vegetables) have a negative effect on muscle mass. ${ }^{31}$ The above diet deficiencies in conjunction with the fact that the elderly tend to take in less calories in general can lead to severe protein deficits as well as deficiencies in other nutritive elements.

It has in general been shown that adequate food intake in elderly people protects them from sarcopenia, also that older adults are likely to need more protein per kilogram than young people in order to preserve adequate protein levels that support muscle mass ${ }^{32,33}$ with about 1.0-1.2 $\mathrm{gr} / \mathrm{kg}$ protein intake per day probably being optimal for this age group.

In conclusion, the maintenance of adequate protein intake is of crucial importance during the treatment of sarcopenia. For this purpose a number of nutritive and dietary supplements can be used. 
There is some evidence suggesting that creatine supplements may help in muscle development in older adults who follow a training program with resistance exercise. ${ }^{34,35}$ Maintaining the right vitamin D blood levels might also help preserve muscle power. In a study concerning clinical interventions in sarcopenia and a healthy appearance among elderly people in developed countries, a strong correlation was found between protein intake per meal and loss of muscle mass. Moreover, it has been noted that apart from an inadequate? dietary protein intake, certain other? adverse factors need to be taken into account: insulin resistance, cytokines, vitamin D deficiency and a sedentary lifestyle. All such data will provide a clearer understanding of the negative changes in body composition as age advances and thereby contribute to the designing of more effective therapeutic interventions. ${ }^{35}$

\section{Physical Activity}

The contribution of exercise, especially resistance exercise (or strengthening exercise), in the prevention of sarcopenia is of substantial importance. Resistance exercises positively impact the neuromuscular system, protein synthesis and the hormones, which cause sarcopenia when they function abnormally. Studies have shown that after a resistance exercise program, the triggering of the motor neurons and of protein synthesis (both essential to build muscle mass) increase, even in the elderly. ${ }^{36,37}$ These changes indicate that the redevelopment of muscle mass is possible even in people of an advanced age.

More specifically, resistance exercise is considered to be a type of "strength" training increases maximum strength since persistent training is thought to enable an individual to progressively lift ever bigger weights. However, taking the physical laws into consideration, the issue is more complicated. Supposing that a $100 \mathrm{~kg}$ object (total weight: $100 \mathrm{~kg} \times 9.81 \mathrm{~m} / \mathrm{s} \approx 1000 \mathrm{~N}$ ) must be lifted in $1 \mathrm{~min}$, the energy that must be produced will be $1000 \mathrm{~N} \times 1 \mathrm{~m}=1000 \mathrm{~J}$. If the above takes place in $1 \mathrm{sec}$, the result is an average power of 1000 $\mathrm{j} / 1 \mathrm{sec}=1000 \mathrm{~W}$, while if it takes place in $0.5 \mathrm{sec}$ the average power is $2000 \mathrm{~W}(1000 \mathrm{j} / 0.5 \mathrm{sec}=2000$ $\mathrm{W})$. To conclude, from the viewpoint of mechanical physics, exercises using weights are intended for increasing power, not force.
Aerobic exercise also appears to help fight sarcopenia. This type of exercise has been shown to help increase protein synthesis, an important function for preservation of muscle mass and strength in the elderly. ${ }^{37}$ Of note, though certainly physical activity improves and even prevents sarcopenia, this must be accompanied by good nutrition.

\section{Drugs}

There is an attempt to design medications to both prevent and treat sarcopenia and indeed numerous pharmaceutical drugs have been tested in various clinical trials.

Theoretically, the ideal drug should be able to achieve increase of muscle and bone tissue mass while simultaneously decreasing fatty tissue. A protein that is being investigated for its effect on muscle tissue mass is myostatin (GDF-8-growth differentiation factor- 8$).^{38}$ Myostatin is produced by myocytes and acts on the autocrine function of muscle cells to inhibit their growth and differentiation, thereby inhibiting the synthesis of muscle tissue. Animals with mutations in the myostatin gene that lead to the inability to produce functioning myostatin and animals treated with substances that block the activity of this protein have significantly more muscle mass (for example, the Belgian Blue cow). Myostatin binds to the ARIIB receptor (activin receptor type IIB) and modifies the metabolic activity and homeostasis of the muscle via three intracellular pathways. ${ }^{39}$ In patients with cachexia due to chronic disease, cancer or acquired immunodeficiency syndrome, increased myostatin levels have been found which is probably responsible for the low muscle mass in these individuals. It is generally known that myostatin levels are related not only to muscle mass but also to functional muscle parameters, such as power and force. Furthermore, myostatin is decreased during exercise and is related to the type of exercise (resistance exercise $>$ endurance exercise), the intensity (light resistance does not modify it, while medium resistance does), as well as the person's potential (inactive individuals tend to show a greater decrease compared to athletes). ${ }^{38}$

ACE-031, a recombined protein that was made from the extracellular part of the ARIIB receptor combined with the $\mathrm{Fc}$ region of human immunoglobin 
IgG1, which acts as a strong myostatin inhibitor, was used during a double blind placebo control study in 48 healthy, post-menopausal women. The drug was well tolerated (with a rash as the only adverse effect), and it achieved a statistically significant increase in muscle mass, while at the same time it improved bone and fat tissue metabolism. ${ }^{40}$

The anabolic hormone testosterone increased muscle mass, power, force and function; however, it caused water retention, an increase in hematocrit, short-term worsening of sleep apnea and an increase in the frequency of cardiovascular accidents, while it also appear to be dangerous in cases of prostatic hypertrophy. Selective androgen receptor modulators (SARMs) have succeeded in augmenting muscle mass with in addition a slight increase in muscle power, but it may worsen existing heart failure (Table). In other studies, the use of growth hormone was related to a positive nitrogen $(\mathrm{N})$ balance and to increase of muscle mass; on the other hand, the patients developed joint pain, muscle pain, edema, carpal tunnel syndrome and hyperglycemia. Ghrelin (a hormone that increases appetite) agonists have been shown to augment muscle mass, however with adverse effects such as fatigue, dyspnea and atrial fibrillation. Antimyostatin antibodies appeared to increase fat-free muscle mass (lean body mass) and grip strength, though the following adverse effects were concurrently reported: allergic urticaria, aseptic meningitis, diarrhea, confusion and fatigue. After using activin type IIR receptor antagonists, there was an increase of muscle volume, as measured in the thigh, of muscle mass and of the distance a person could walk in 6 minutes, while the adverse effects included acne

Table. Selective androgen receptor modulators (SARMs) pre-clinical and clinical development

\begin{tabular}{|c|c|c|c|}
\hline Drug & $\begin{array}{l}\text { Commercial } \\
\text { name }\end{array}$ & Status of study & Indication \\
\hline $\begin{array}{l}\text { MK2866 } \\
\text { GTx-024 }\end{array}$ & $\begin{array}{l}\text { Ostarine } \\
\text { Enobosarm }\end{array}$ & $\begin{array}{l}\text { Phase II b } \\
\text { Clinical study }\end{array}$ & $\begin{array}{l}\text { Increase of } \\
\text { muscle mass }\end{array}$ \\
\hline LGD-4033 & Ligandrol & $\begin{array}{l}\text { Pre-clinical } \\
\text { study }\end{array}$ & $\begin{array}{l}\text { Improves } \\
\text { muscle mass }\end{array}$ \\
\hline 971086 & GSK 971086 & $\begin{array}{l}\text { Clinical study } \\
\text { phase I (2011) }\end{array}$ & $\begin{array}{l}\text { Androgen } \\
\text { agonist }\end{array}$ \\
\hline Karo & & Stopped & \\
\hline
\end{tabular}

and involuntary muscle contractions (cramps). Perindropril, an angiotensin-converting-enzyme inhibitor, increased the distance that the patient could walk and decreased hip fracture frequency, although it caused hypotension, hyperkalemia, muscle cramps and numbness. The two latest drugs that have been tested are Pindolol, a non-selective beta blocker (antagonist) was able to preserve muscle mass and increased grip strength in the elderly, and Tirasemtiv, which seemed to improve muscle function. No significant adverse effects have been reported. ${ }^{41}$

\section{REFERENCES}

1. Rodriguez-Mañas L, Rodríguez-Artalejo F, Sinclair AJ, 2017 The Third Transition: The Clinical Evolution Oriented to the Contemporary Older Patient. J Am Med Dir Assoc 18: 8-9.

2. Brink W, 2007 Preventing Sarcopenia. LifeExtension Magazine.

3. Anker SD, Morley JE, von Haehling S, 2016 Welcome to the ICD-10 code for sarcopenia. J Cachexia Sarcopenia Muscle 7: 512-514.

4. Working Group on Functional Outcome Measures for Clinical Trials, Bhasin S, Espeland MA, Evans WJ, et al, 2009 Indications, labeling, and outcomes assessment for drugs aimed at improving functional status in older persons: a conversation between aging researchers and FDA regulators. J Gerontol A Biol Sci Med Sci 64: 487-491.

5. Rosenberg I, 1989 Summary comments: epidemiological and methodological problems in determining nutritional status of older persons. Am J Clin Nutr 50: 1231-1233.

6. Castillo EM, Goodman-Gruen D, Kritz-Silverstein D, Morton DJ, Wingard DL, Barrett-Connor E, 2003 Sarcopenia in elderly men and women - the Rancho Bernardo study. Am J Prev Med 25: 226-231.

7. Mithal A, Bonjour JP, Boonen S, et al, 2013 IOF CSA Nutrition Working Group. Impact of nutrition on muscle mass, strength, and performance in older adults. Osteoporos Int 24: 1555-1566.

8. Cruz-Jentoft AJ, Baeyens JP, Bauer JM, et al, European Working Group on Sarcopenia in Older People 2010, Sarcopenia: European consensus on definition and diagnosis: Report of the European Working Group on Sarcopenia in Older People. Age Ageing 39: 412-423.

9. Janssen I, Heymsfield SB, Ross R, 2002 Low relative skeletal muscle mass (sarcopenia) in older persons is associated with functional impairment and physical disability. J Am Geriatr Soc 50: 889-896.

10. Yaxley A, Miller MD, Fraser RJ, Cobiac L, Crotty M, 2012 The complexity of treating wasting in ambulatory rehabilitation: Is it starvation, sarcopenia, cachexia or a 
combination of these conditions? Asia Pac J Clin Nutr 21: 386-393.

11. Janssen I, Shepard DS, Katzmarzyk PT, Roubenoff R, 2004 The healthcare costs of sarcopenia in the United States. J Am Geriatr Soc 52: 80-85.

12. Baumgartner RN, Koehler KM, Gallagher D, et al, 1998 Epidemiology of sarcopenia among the elderly in New Mexico. Am J Epidemiol 147: 755-763.

13. Cruz-Jentoft AJ, Landi F, Schneider SM, et al, 2014 Prevalence of and interventions for sarcopenia in ageing adults: a systematic review. Report of the International Sarcopenia Initiative (EWGSOP and IWGS). Age Ageing 43: 748-759.

14. Fielding RA, Vellas B, Evans WJ, et al, 2011 Sarcopenia: an undiagnosed condition in older adults. Current consensus definition: prevalence, etiology, and consequences. International working group on sarcopenia. J Am Med Dir Assoc 12: 249-256.

15. Joseph C, Kenny AM, Taxel P, Lorenzo JA, Duque G, Kuchel GA, 2005 Role of endocrine-immune dysregulation in osteoporosis, sarcopenia, frailty and fracture risk. Mol Aspects Med 26: 181-201.

16. Lang T, Streeper T, Cawthon P, Baldwin K, Taaffe DR, Harris TB, 2010 Sarcopenia: etiology, clinical consequences, intervention, and assessment. Osteoporos Int 21: 543-559.

17. Zisman A, Peroni OD, Abel ED, et al, 2000 Targeted disruption of the glucose transporter 4 selectively in muscle causes insulin resistance and glucose intolerance. Nat Med 6: 924-928.

18. Hedman A, Berglund L, Essén-Gustavsson B, Reneland R, Lithell H, 2000 Relationships between muscle morphology and insulin sensitivity are improved after adjustment for intra-individual variability in 70-year-old men. Acta Physiol Scand 169: 125-132.

19. Johnston DE, 1985 Tendons, skeletal muscles, and ligaments in health and disease. In: Newton CD, Nunamaker DM, Textbook of small animal orthopaedics. Philadelphia: Lippincott, pp: 65-76.

20. Patel HP, Clift E, Lewis L, Cooper C, 2017 Epidemiology of sarcopenia and frailty, frailty and sarcopenia - Onset, development and clinical challenges, Yannis Dionyssiotis (Ed.), InTech, DOI: 10.5772/intechopen.69771. Available from: https:/www.intechopen.com/books/ frailty-and-sarcopenia-onset-development-and-clinicalchallenges/epidemiology-of-sarcopenia-and-frailty

21. Kim J, Wang Z, Heymsfield SB, Baumgartner RN, Gallagher D, 2002 Total-body skeletal muscle mass: estimation by a new dual-energy X-ray absorptiometry method. Am J Clin Nutr 76: 378-383.

22. Clark BC, Manini TM, 2008 Sarcopenia =/=dynapenia. J Gerontol A Biol Sci Med Sci 63: 829-834.

23. Peeters GM, de Vries OJ, Elders PJ, Pluijm SM, Bouter LM, Lips P, 2007 Prevention of fall incidents in patients with a high risk of falling: design of a randomised controlled trial with an economic evaluation of the effect of multidisciplinary transmural care. BMC Geriatr 7: 15 .

24. Runge M, Schacht E, 2005 Multifactorial pathogenesis of falls as a basis for multifactorial interventions. $\mathrm{J}$ Musculoskelet Neuronal Interact 5: 127-134.

25. Lauretani F, Russo CR, Bandinelli S, et al, 2003 Ageassociated changes in skeletal muscles and their effect on mobility: an operational diagnosis of sarcopenia. J Appl Physiol (1985) 95: 1851-1860.

26. Ilie AC, Pislaru AI, Macsim IC, 2017 Sarcopenia and the importance of biochemical determinations in the hospitalised older adults. E-Health and Bioengineering Conference (EHB), Sinaia, pp; 281-284.

27. Volpi E, Nazemi R, Fujita S, 2004 Muscle tissue changes with aging. Curr Opin Clin Nutr Metab Care 7: 405-410.

28. Dionyssiotis Y, Chhetri JK, Piotrowicz K, Gueye T, Sánchez E, 2017 Impact of nutrition for rehabilitation of older patients: report on the 1st EICA-ESPRM-EUGMS train the trainers course. Eur Geriatr Med 8: 183-190.

29. Waters DL, Baumgartner RN, Garry PJ, 2000 Sarcopenia: current perspectives. The Journal of Nutrition Health and Aging 4: 133-139.

30. Houston DK, Nicklas BJ, Ding J, et al, 2008 Dietary protein intake is associated with lean mass change in older, community-dwelling adults: the Health, Aging, and Body Composition (Health ABC) Study. Am J Clin Nutr January 87: 150-155.

31. Campbell WW, Crim MC, Dallal GE, Young VR, Evans WJ, 1994 Increased protein requirements in elderly people: new data and retrospective reassessment. Am J Clin Nutr 60: 501-509.

32. Campbell WW, Evans WJ, 1996 Protein requirements of elderly people. Eur J Clin Nutr 50: Suppl 1: 180-185.

33. Brose A, Parise G, Tarnopolsky MA, 2003 Creatine supplementation enhances isometric strength and body composition improvements following strength exercise training in older adults. J Gerontol A Biol Sci Med Sci 58: 11-19.

34. Chrusch MJ, Chilibeck PD, Chad KE, Davison KS, Burke DG, 2001 Creatine supplementation combined with resistance training in older men. Med Sci Sports Exerc 33: 2111-2117.

35. Valenzuela RE, Ponce JA, Morales-Figueroa GG, Muro KA, Carreón VR, Alemán-Mateo H, 2013 Insufficient amounts and inadequate distribution of dietary protein intake in apparently healthy older adults in a developing country: implications for dietary strategies to prevent sarcopenia. Clin Interv Aging 8: 1443-1148.

36. Roth SM, Ferrel RE, Hurley BF, 2000 Strength Training for the Prevention and Treatment of Sarcopenia. J Nutr Health Aging 4: 143-155.

37. Hasten DL, Pak-Loduca J, Obert KA, Yarasheski KE, 2000 Resistance exercise acutely increases MHC and 
mixed muscle protein synthesis rates in 78-84 and 23-32 yr olds. Am J Physiol Endocrinol Metab 278: E620-626.

38. Buehring B, Binkley N, 2013 Myostatin--the holy grail for muscle, bone, and fat? Curr Osteoporos Rep 11: 407-414.

39. Elliott B, Renshaw D, Getting S, Mackenzie R, 2012 The central role of myostatin in skeletal muscle and whole body homeostasis. Acta Physiol (Oxf) 205: 324-340.

40. Attie KM, Borgstein NG, Yang Y, et al, 2013 A single ascending-dose study of muscle regulator ACE-031 in healthy volunteers. Muscle Nerve 47: 416-423.

41. Meriggioli MN, Roubenoff R, 2015 Prospect for pharmacological therapies to treat skeletal muscle dysfunction. Calcif Tissue Int 96: 234-242. 